Check for updates

Cite this: Mater. Chem. Front., 2019, 3, 2746

Received 18th September 2019, Accepted 10th October 2019

DOI: $10.1039 / c 9 q m 00586 b$

rsc.li/frontiers-materials

\section{Utilizing the aggregation-induced emission phenomenon to visualize spontaneous molecular directed motion in the solid state $\uparrow$}

\author{
Jianxun Liu, $\ddagger^{a}$ Chang Xing, $\ddagger^{a}$ Donghui Wei, (D) a Qianqian Deng, ${ }^{a}$ Cuiping Yang, ${ }^{a}$ \\ Qiuchen Peng, ${ }^{\mathrm{ab}}$ Hongwei Hou, (D) *a Yuanyuan $\mathrm{Li}^{\mathrm{b}}$ and Kai $\mathrm{Li}$ (D) *a
}

\begin{abstract}
The real-time monitoring of spontaneous molecular directed motion is a highly important but very challenging task. In this work, a rod-like aggregation-induced emission (AIE) molecule was carefully designed and facilely synthesized. The AIE molecule, the salicylaldehyde 4-butoxyaniline Schiff base (SBA), exhibited a unique self-recovery property from a semi-ordered structure to an ordered structure along with significant fluorescence changes after grinding. The fluorescence changes were monitored to obtain important kinetic information regarding the spontaneous molecular directed motion process, including the kinetic order, rate constants, half-life, and apparent activation energy. Unlike instrumental analytical methods such as PXRD and AFM, which only give information on a stable state of samples, the proposed fluorescence method provides a new perspective for real-time visualization of spontaneous molecular directed motion in situ in the solid state.
\end{abstract}

\section{Introduction}

Spontaneous molecular directed motions induced by intermolecular interactions such as hydrogen bonding, $\pi-\pi$ interactions, $\mathrm{C}-\mathrm{H} \cdots \pi$ interactions and hydrophobic interactions are an important driving force for molecular self-assembly, and are ubiquitous in various biochemical pathways such as DNA/RNA pairing, enzyme folding/unfolding, membrane transport and molecular recognition. ${ }^{1-6}$ Thus, the observation and measurement of spontaneous molecular directed motion are important for revealing life processes. However, due to the random nature of molecular motions, their characterization remains a challenging task.

In recent years, monitoring changes in fluorescence has been demonstrated to be an effective method to visualize various chemical and physical processes. ${ }^{7-9}$ For example, small molecule aggregation processes, the macromolecule cononsolvency effect, polymer glass transitions and gelation processes have been

\footnotetext{
${ }^{a}$ College of Chemistry and Molecular Engineering, Zhengzhou University,

Henan 450001, P. R. China. E-mail: likai@zzu.edu.cn

${ }^{b}$ College of Chemistry, Chemical and Environmental Engineering,

Henan University of Technology, Henan 450001, P. R. China

$\dagger$ Electronic supplementary information (ESI) available: Details on experimental procedures. A video for the self-recovery process of SBA. Additional experimental data, including crystal data, ${ }^{1} \mathrm{H}$-NMR spectra, ${ }^{13} \mathrm{C}$-NMR spectra, mass spectrometry, etc. CCDC 1941969-1941971. For ESI and crystallographic data in CIF or other electronic format see DOI: 10.1039/c9qm00586b

\$ J. Liu and C. Xing contributed equally.
}

successfully visualized in real-time in situ by using different luminescent molecules. ${ }^{10-13}$ Recently, Prof. Tang and co-workers designed and synthesized a new aggregation-induced emission (AIE) molecule with self-recovery properties. ${ }^{14}$ Due to the intense and adjustable luminescence property of the AIE molecule in the solid state, the spontaneous molecular motion of the AIE molecule from the amorphous state to the crystal state induced by $\pi-\pi$ interactions was visualized. However, the molecular motion is random and nondirected, making it difficult to fully understand and characterize it. Especially, kinetic information of the molecular motion was barely investigated. To date, the visualization of spontaneous molecular directed motion in the solid state is still not realized.

In order to visualize spontaneous molecular directed motions in the solid state, a new AIE molecule, the salicylaldehyde 4-butoxyaniline Schiff base (SBA), was deliberately prepared in this work. SBA was designed to consist of two parts, a salicylaldehyde aniline Schiff base part and a butoxy part, as illustrated in scheme 1. The salicylaldehyde aniline Schiff base is a typical AIE group, which is expected to endow SBA with visible luminescence changes after stimulating with mechanical force such as grinding. ${ }^{15-18}$ The butoxy group has strong hydrophobic interactions, and can facilitate the selfrecovery of SBA in the solid state. ${ }^{19-21}$ Moreover, inspired by the design strategy of rod-like liquid crystal molecules which can undergo directional alignment in the mesogenic state, the butoxy group was attached in the para-position of the $\mathrm{N}$ atom, resulting in a rod-like structure for SBA. This structural 


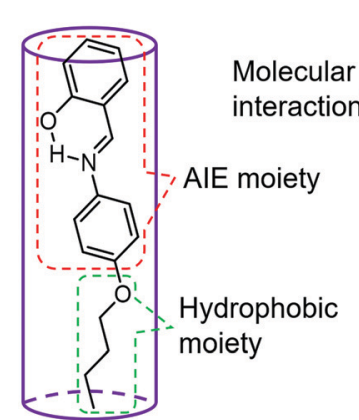

Rod-like structure

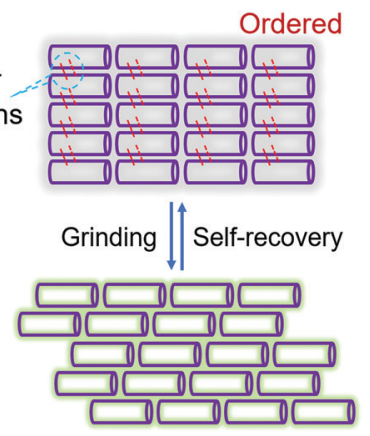

Semi-ordered
Scheme 1 Design strategy of SBA.

feature is expected to help SBA maintain a semi-ordered spatial arrangement even after grinding.

\section{Results and discussion}

The AIE property of SBA was first investigated in EtOH/ $\mathrm{H}_{2} \mathrm{O}$ mixtures with different water fractions $\left(f_{\mathrm{w}}\right)$. As shown in Fig. 1A and B, SBA exhibited weak fluorescence in the good solvent of EtOH but intense fluorescence in the poor solvent of $\mathrm{H}_{2} \mathrm{O}$, suggesting its AIE feature. ${ }^{2-25}$ The aggregation of SBA in $\mathrm{H}_{2} \mathrm{O}$ was confirmed by absorption spectra and dynamic light scattering (DLS). In EtOH, SBA exhibited flat baselines in the absorption spectrum over $400 \mathrm{~nm}$. In contrast, a level-off tail in the absorption spectrum was observed in $\mathrm{H}_{2} \mathrm{O}$, which could be attributed to the light scattering of the aggregated suspensions (Fig. 1C). ${ }^{26-28}$ Moreover, DLS results suggested that nano-particles with a diameter around $500 \mathrm{~nm}$ existed in $\mathrm{H}_{2} \mathrm{O}$ but no particles were observed in EtOH (Fig. 1D). The above results demonstrated that
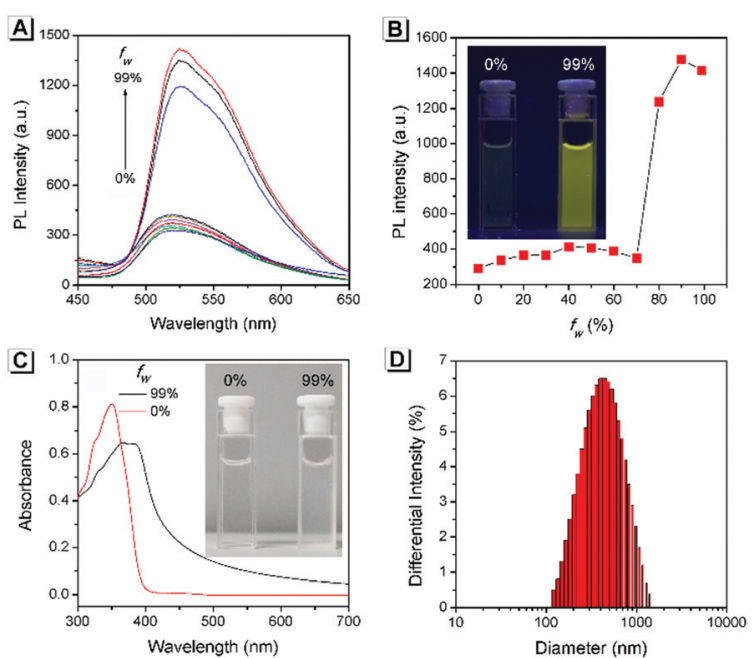

Fig. 1 (A) Fluorescence spectra of SBA in water/ethanol mixtures with different $f_{\mathrm{w}}$. (B) Fluorescence intensity of SBA at $525 \mathrm{~nm}$ as a function of $f_{\mathrm{w}}$. (C) Absorption spectra of SBA in water/ethanol mixtures with $f_{w}$ of $0 \%$ and $99 \%$. (D) DLS result of SBA in water/ethanol mixtures with an $f_{w}$ of $99 \%$. Inset: A photo of SBA in water/ethanol mixtures with $f_{\mathrm{w}}$ of $0 \%$ and $99 \%$; (B) under UV light irradiation and (C) under daylight.
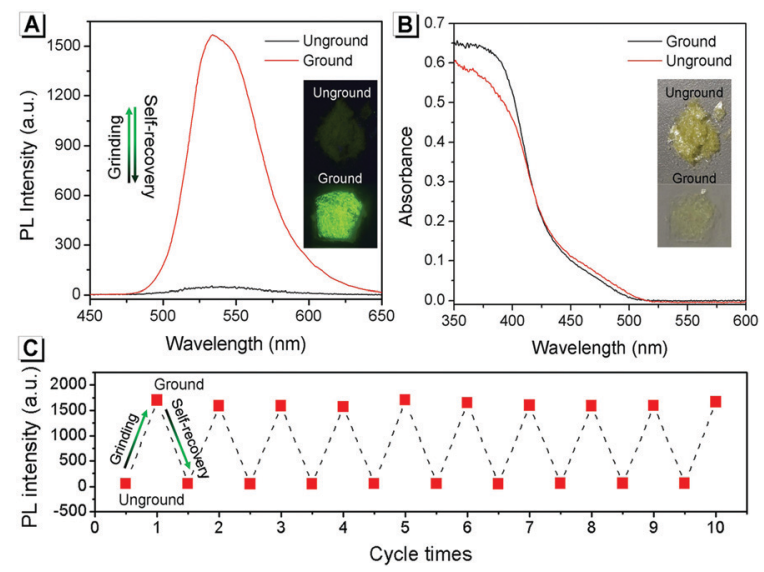

Fig. 2 (A) Fluorescence and (B) UV-vis diffuse reflectance spectra of SBA before and after grinding. (C) Fatigue resistance of the fluorescence intensity of SBA during 10 grinding/self-recovery cycles. All spectra were recorded at $0{ }^{\circ} \mathrm{C}$.

the intense fluorescence of SBA in aqueous solution originated from aggregation, i.e., AIE fluorescence.

The self-recovery property of SBA in the solid state can be visually observed through the change in its fluorescence. As shown in Fig. 2A, SBA exhibited almost no fluorescence in the solid state. After grinding, intense but transitory green emission around $525 \mathrm{~nm}$ was observed, which disappeared within $1 \mathrm{~min}$ at room temperature. Meanwhile, no obvious changes were observed in the UV-vis diffuse reflectance spectra, suggesting that no new substance was formed after grinding (Fig. 2B). The emission turn-on property by grinding and fast self-recovery was highly reversible. As shown in Fig. 2C, after repeatedly toggling between the ground and unground states (10 times), the fluorescence of SBA at $525 \mathrm{~nm}$ remained almost constant without degradation, indicating its good fatigue resistance.

To understand these phenomena, single-crystal X-ray diffraction (SCXRD) analysis was performed. The results confirmed that SBA was a rod-like molecule in the crystal, and the dihedral angle between two conjugate planes was $49.0^{\circ}$ (Fig. 3A). The molecules were distributed in layers. Inside the layers, the molecules were arranged in "head-to-head" and "tail-to-tail" fashion (Fig. 3B and C). The minimum distance between a hydrogen atom and an adjacent benzene ring in another layer was about $2.9 \AA$, indicating that strong $\mathrm{C}-\mathrm{H} \cdots \pi$ interactions existed between the layers (generally, the necessary

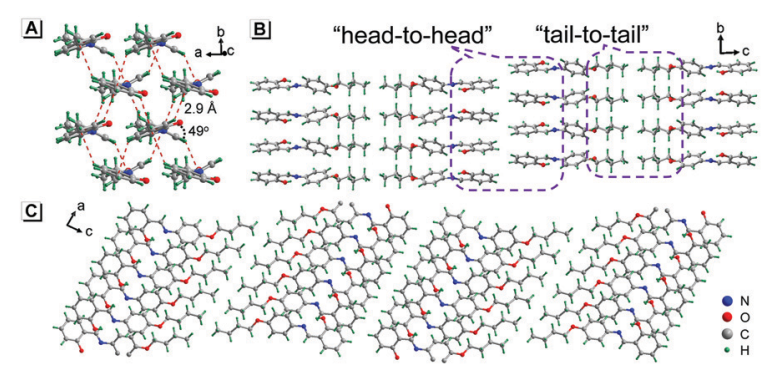

Fig. 3 Crystal structure of SBA viewed from different directions. 
condition for $\mathrm{C}-\mathrm{H} \cdots \pi$ interactions is that the distance between the hydrogen atom and the adjacent benzene ring is smaller than $3.0 \AA^{29-31}$ ). The existence of $\mathrm{C}-\mathrm{H} \cdots \pi$ interactions possibly facilitates the non-radiative transition of electrons in the excited state, which might be the primary reason for the fluorescence quenching in the crystal state. Under mechanical force, the characteristic stacked structure and $\mathrm{C}-\mathrm{H} \cdots \pi$ interactions were destroyed, resulting in intense green emission. This proposed mechanism for the fluorescence turn-on of SBA after grinding is shown in Scheme 1.

To provide support for this proposed mechanism, contrast experiments were carried out. Two control compounds were synthesized having shorter carbon chains compared to SBA, namely the salicylaldehyde 4-ethyoxyaniline Schiff base (SEA) and the salicylaldehyde 4-propoxyaniline Schiff base (SPA) (Scheme S1, ESI $\dagger$ ). The crystal structures of SEA (Fig. S2, ESI $\dagger$ ) and SPA (Fig. S3, ESI $\dagger$ ) were very similar to that of SBA, and the SEA and SPA molecules were also arranged head-to-head and tail-to-tail in layers in their crystals, respectively. However, the fluorescence changes of SEA and SPA before and after grinding were quite different compared to SBA (Fig. 4A). For SEA, there was barely any change in emission after grinding. For SPA, only 1.5-fold emission enhancement was observed after grinding. For SBA, the emission enhancement after grinding was as high as 35-fold compared to SBA without grinding. These results confirmed that shorter carbon chains could not effectively insulate the salicylaldehyde aniline Schiff base moieties in adjacent molecules after grinding, resulting in weaker fluorescence changes (Fig. 4B).

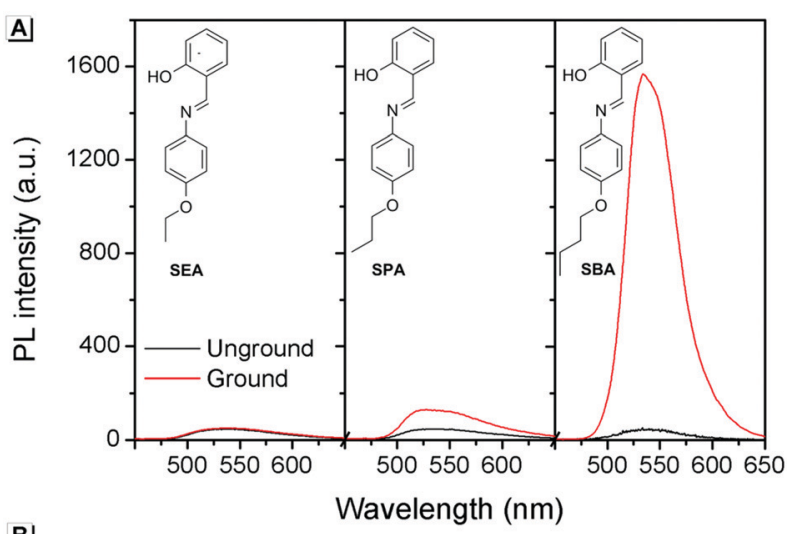

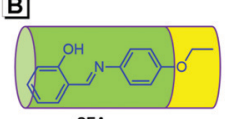

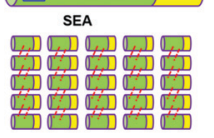

Grinding $\mid \rrbracket$ Self-recovery

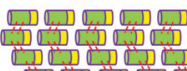

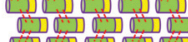

(No fluorescence)
(rong molection interaction

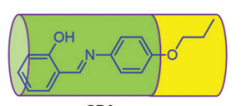

SPA
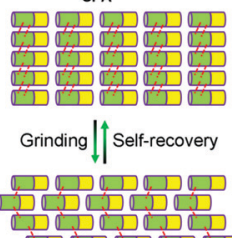

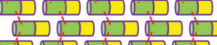

Weak molecular interactions (Weak fluorescence)
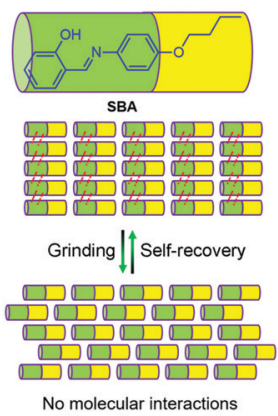

No molecular interactions (Strong fluorescence)
Fig. 4 (A) Fluorescence spectra of SEA, SPA and SBA before and after grinding. (B) Proposed mechanism for the fluorescence changes.
Furthermore, PXRD measurements were carried out at $0{ }^{\circ} \mathrm{C}$ to investigate the structural changes in SBA before and after grinding. At this temperature, the fluorescence emission of ground SBA is relatively stable (the temperature-dependent recovery rate will be discussed in detail later). As shown in Fig. 5A, the PXRD patterns of unground SBA fitted well with the simulated one, demonstrating its uniform crystal state. Surprisingly, although most of the diffraction peaks decreased and even disappeared after grinding, some peaks still remained, which indicated that SBA was not completely converted from the crystal to the amorphous form. After analysis and comparison, it was confirmed that these remaining diffraction peaks belonged to the $(0,0, n)$ crystal plane, indicating that the molecular arrangement was still ordered along the $c$-axis after grinding (i.e., a semiordered structure). More importantly, when the ground SBA was kept in the dark at room temperature, all the original diffraction peaks reappeared gradually, which suggested that the ordered crystal structure of SBA was recovered. ${ }^{32-34}$ This unique selfrecovery process from semi-ordered structure to ordered structure along with fluorescence changes provides a visual prototype to understand spontaneous molecular directed motion in the solid state.

Atomic force microscopy (AFM) was used to investigate the change in morphology of SBA in different states. As shown in Fig. 5B, splintery patches were observed in the luminescent state of SBA after grinding. After tuning back to the nonluminescent state, the splintery patches disappeared and larger patches were observed in the same area (Fig. 5C). The AFM patterns suggested that the morphology of SBA became more orderly after self-recovery in the dark, which was as expected.

The above PXRD and AFM analyses provided useful information about the stable state of SBA, while real-time information for SBA can be obtained using the fluorescence method. Thus, time-dependent fluorescence spectra were recorded at different temperatures and kinetic analyses were carried out, which offered rich kinetic information about the spontaneous molecular directed motion. As shown in Fig. 6A, the luminescence intensity decay at $525 \mathrm{~nm}$ can be fitted well with first-order reaction kinetics ( $\ln F$ is proportional to $t$, where $t$ is time and $F$ is the emission intensity at $525 \mathrm{~nm}$ ), suggesting that the spontaneous molecular directed motion of ground SBA was a first-order dynamic process. The rate constant $(k)$ and half-life
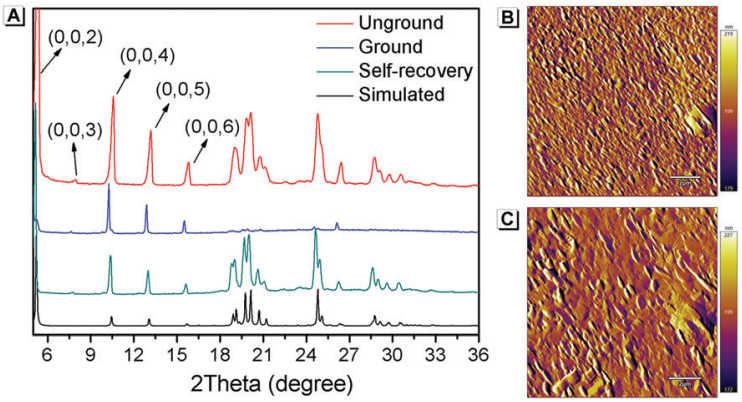

Fig. 5 (A) PXRD spectra of SBA in different states. AFM patterns of ground SBA (B) before and (C) after self-recovery. The scale bar is $2 \mu \mathrm{m}$. 

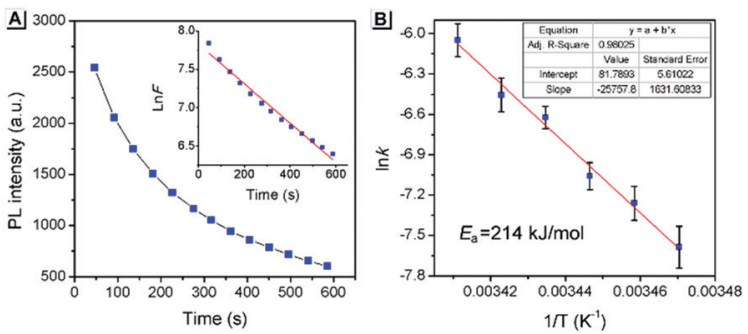

Fig. 6 (A) The thermal fading kinetics of ground SBA at $20{ }^{\circ} \mathrm{C}$. Inset: The curves fitted with first-order reaction dynamics. (B) The rate constants obtained from different temperatures fitted with Arrhenius expressions. The inset shows the fitting parameters.

of ground SBA $\left(t_{1 / 2}\right)$ were calculated according to the integral rate equation of a first-order reaction:

$$
\begin{gathered}
-k t=\ln F \\
t_{1 / 2}=\ln 2 / k
\end{gathered}
$$

The calculated values of $k$ and $t_{1 / 2}$ from $15{ }^{\circ} \mathrm{C}$ to $20{ }^{\circ} \mathrm{C}$ are shown in Table S1 (ESI $\dagger$ ). Based on these data, the apparent activation energy $\left(E_{\mathrm{a}}\right)$ of spontaneous molecular directed motion from the semi-ordered structure to the ordered structure was evaluated according to the Arrhenius expression:

$$
\ln k=-E_{\mathrm{a}} / R T+B
$$

where $T$ is the temperature, $R$ is the ideal gas constant and $B$ is a constant. As shown in Fig. 6B, the apparent $E_{\text {a }}$ was calculated to be $214 \mathrm{~kJ} \mathrm{~mol}^{-1}$, which is higher than most room-temperature spontaneous chemical reactions. These results indicate that intermolecular interactions provided a strong driving force for spontaneous molecular directed motion of SBA, which can overcome the large activation barrier. Moreover, according to the Arrhenius expression, the large apparent $E_{\mathrm{a}}$ suggests that the spontaneous molecular directed motion can be effectively controlled by temperature. As shown in Fig. 7, the green emission of ground SBA was quenched quickly at $25{ }^{\circ} \mathrm{C}$, and it almost disappeared after keeping in the dark for $1 \mathrm{~min}$. In contrast, the green emission of ground SBA barely changed at $0{ }^{\circ} \mathrm{C}$ when it was kept in the dark for $20 \mathrm{~min}$.

Furthermore, density functional theory (DFT) calculations and non-covalent interaction (NCI) analysis were performed to determine the binding energy of the molecules. The single crystal was used as the initial geometry for structural optimization. As

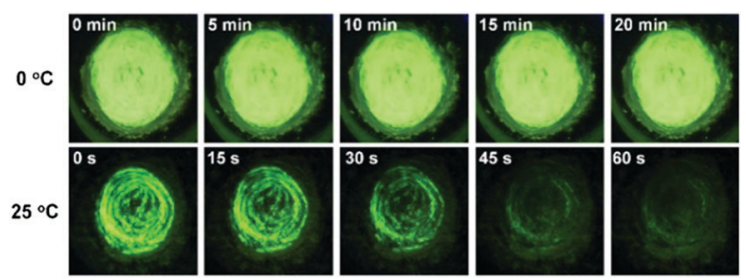

Fig. 7 Photo of ground SBA kept in the dark at $0{ }^{\circ} \mathrm{C}$ and $25^{\circ} \mathrm{C}$.

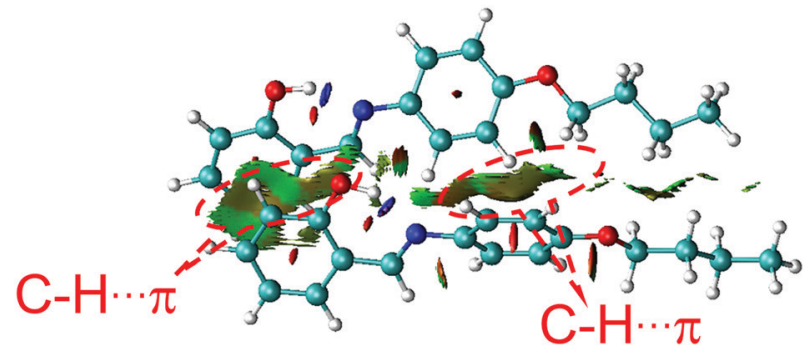

Fig. 8 The $\mathrm{NCl}$ analysis of SBA.

shown in Fig. 8 and Fig. S4 (ESI $\dagger$ ), the calculated binding energy was $52.7 \mathrm{~kJ} \mathrm{~mol}^{-1}$, which was mainly contributed by the $\mathrm{C}-\mathrm{H} \cdots \pi$ interactions between two adjacent molecules. In addition, it can be seen from the crystal structure (Fig. 3A) that each SBA molecule was surrounded by four other SBA molecules. Therefore, the total binding energy is $4 \times 52.7 \mathrm{~kJ} \mathrm{~mol}^{-1}$, i.e., $211 \mathrm{~kJ} \mathrm{~mol}^{-1}$, which is in good agreement with the measured apparent $E_{\mathrm{a}}$ of spontaneous molecular directed motion from the semi-ordered structure to the ordered structure. Thus, it can be inferred that $\mathrm{C}-\mathrm{H} \cdots \pi$ interactions between adjacent molecules are the main driving force for spontaneous molecular directed motion.

\section{Conclusions}

In this work, a rod-like AIE molecule of SBA was prepared, which exhibited unique self-recovery property with significant fluorescence changes after grinding in the solid state. PXRD measurements indicated that there was a directed motion of SBA molecules from a semi-ordered structure to an ordered structure. The fluorescence changes were monitored, which provided rich kinetic information regarding the spontaneous molecular directed motion process, including the kinetic order, rate constants, half-life and apparent activation energy. Furthermore, DFT calculations and NCI analysis were performed, which demonstrated that the $\mathrm{C}-\mathrm{H} \cdots \pi$ interactions between adjacent molecules were the main driving force for the spontaneous molecular directed motion of SBA. Unlike instrumental analytical methods such as PXRD and AFM, which only provide information about the stable state of samples, the proposed fluorescence method offers a new strategy for real-time visualization of spontaneous molecular directed motion in situ in the solid state.

\section{Conflicts of interest}

There are no conflicts to declare.

\section{Acknowledgements}

This work was financially supported by the National Natural Science Foundation of China (No. 21501150, 51502079, 21671174) and Zhongyuan thousand talents project. 


\section{Notes and references}

1 K. L. Hudson, G. J. Bartlett, R. C. Diehl, J. Agirre, T. Gallagher, L. L. Kiessling and D. N. Woolfson, J. Am. Chem. Soc., 2015, 137, 15152-15160.

2 M. Harigai, M. Kataoka and Y. Imamoto, J. Am. Chem. Soc., 2006, 128, 10646-10647.

3 M. del Carmen Fernandez-Alonso, F. J. Canada, J. JimenezBarbero and G. Cuevas, J. Am. Chem. Soc., 2005, 127, 7379-7386.

4 L. M. Salonen, M. Ellermann and F. Diederich, Angew. Chem., Int. Ed., 2011, 50, 4808-4842.

5 M. Minuth and C. Richert, Angew. Chem., Int. Ed., 2013, 52, 10874-10877.

6 R. W. Newberry and R. T. Raines, ACS Chem. Biol., 2019, 14, 1677-1686.

7 N. Jiang, T. Shen, J. Z. Sun and B. Z. Tang, Sci. China Mater., 2019, 62, 1227-1235.

8 H. Zhang, J. Z. Sun, J. Liu, R. T. K. Kwok, J. W. Y. Lam and B. Z. Tang, J. Appl. Phys., 2019, 126, 050901.

9 Y. Li, S. Liu, T. Han, H. Zhang, C. Chuah, R. T. K. Kwok, J. W. Y. Lam and B. Z. Tang, Mater. Chem. Front., 2019, 3, 2207-2220.

10 H. Zhang, X. Zheng, R. T. K. Kwok, J. Wang, N. L. C. Leung, L. Shi, J. Z. Sun, Z. Tang, J. W. Y. Lam, A. Qin and B. Z. Tang, Nat. Commun., 2018, 9, 4961.

11 Z. Wang, J. Nie, W. Qin, Q. Hu and B. Z. Tang, Nat. Commun., 2016, 7, 12033.

12 J. Xue, W. Bai, H. Duan, J. Nie, B. Du, J. Z. Sun and B. Z. Tang, Macromolecules, 2018, 51, 5762-5772.

13 Z. Qiu, E. K. K. Chu, M. Jiang, C. Gui, N. Xie, W. Qin, P. Alam, R. T. K. Kwok, J. W. Y. Lam and B. Z. Tang, Macromolecules, 2017, 50, 7620-7627.

14 P. Alam, N. L. C. Leung, Y. Cheng, H. Zhang, J. Liu, W. Wu, R. T. K. Kwok, J. W. Y. Lam, H. H. Y. Sung, I. D. Williams and B. Z. Tang, Angew. Chem., Int. Ed., 2019, 58, 4536-4540.

15 W. Tang, Y. Xiang and A. Tong, J. Org. Chem., 2009, 74, 2163-2166.

16 P. Song, X. Chen, Y. Xiang, L. Huang, Z. Zhou, R. Wei and A. Tong, J. Mater. Chem., 2011, 21, 13470-13475.
17 Q. Feng, Y. Li, L. Wang, C. Li, J. Wang, Y. Liu, K. Li and H. Hou, Chem. Commun., 2016, 52, 3123-3126.

18 X. Zhang, J. Shi, G. Shen, F. Gou, J. Cheng, X. Zhou and H. Xiang, Mater. Chem. Front., 2017, 1, 1041-1050.

19 X. Zhang, Z. Chi, J. Zhang, H. Li, B. Xu, X. Li, S. Liu, Y. Zhang and J. Xu, J. Phys. Chem. B, 2011, 115, 7606-7611.

20 S. Xue, X. Qiu, Q. Sun and W. Yang, J. Mater. Chem. C, 2016, 4, 1568-1578.

21 Y. Seong-Jun, C. Jong Won, G. Johannes, K. Kil Suk, C. Moon-Gun, K. Dongho and P. Soo Young, J. Am. Chem. Soc., 2010, 132, 13675-13683.

22 Y. Hong, J. W. Y. Lam and B. Z. Tang, Chem. Soc. Rev., 2011, 40, 5361-5388.

23 Y. L. Wang, C. Fan, B. Xin, J. P. Zhang, T. Luo, Z. Q. Chen, Q. Y. Zhou, Q. Yu, X. N. Li, Z. L. Huang, C. Li, M. Q. Zhu and B. Z. Tang, Mater. Chem. Front., 2018, 2, 1554-1562.

24 J. Mei, N. L. C. Leung, R. T. K. Kwok, J. W. Y. Lam and B. Z. Tang, Chem. Rev., 2015, 115, 11718-11940.

25 J. Mei, Y. Hong, J. W. Lam, A. Qin, Y. Tang and B. Z. Tang, Adv. Mater., 2014, 26, 5429-5479.

26 K. Li, Y. Liu, Y. Li, Q. Feng, H. Hou and B. Z. Tang, Chem. Sci., 2017, 8, 7258-7267.

27 J. Chen, C. C. W. Law, J. W. Y. Lam, Y. Dong, S. M. F. Lo, I. D. Williams, D. Zhu and B. Z. Tang, Chem. Mater., 2003, 15, 1535-1546.

28 Y. He, Y. Li, H. Su, Y. Si, Y. Liu, Q. Peng, J. He, H. Hou and K. Li, Mater. Chem. Front., 2019, 3, 50-56.

29 M. Nishio, Phys. Chem. Chem. Phys., 2011, 13, 13873-13900. 30 C. Zhao, R. M. Parrish, M. D. Smith, P. J. Pellechia, C. D. Sherrill and K. D. Shimizu, J. Am. Chem. Soc., 2012, 134, 14306-14309.

31 O. Takahashi, Y. Kohno and M. Nishio, Chem. Rev., 2010, 110, 6049-6076.

32 G. Zhang, J. Lu, M. Sabat and C. L. Fraser, J. Am. Chem. Soc., 2010, 132, 2160-2162.

33 J. Wang, J. Mei, R. Hu, J. Z. Sun, A. Qin and B. Z. Tang, J. Am. Chem. Soc., 2012, 134, 9956-9966.

34 Z. Yang, Z. Chi, Z. Mao, Y. Zhang, S. Liu, J. Zhao, M. P. Aldred and Z. Chi, Mater. Chem. Front., 2018, 2, 861-890. 